\title{
Effects of phase transitions in devices actuated by the electro- magnetic vacuum force
}

\author{
A. Benassi ${ }^{1,2}$ and C. Calandra ${ }^{2}$ \\ 1 CNR/INFM-National Research Center on nanoStructures and bioSystems at Surfaces (S3) \\ Via Campi 213/A, I-41100 Modena, Italy \\ 2 Dipartimento di Fisica, Universitá di Modena e Reggio Emilia \\ Via Campi 213/A, I-41100 Modena, Italy
}

PACS 12.20.Ds - QED specific calculations

PACS $85.85 .+j-$ MEMS/NEMS

PACS 78.66. Jg - Amorphous semiconductors, glasses

\begin{abstract}
We study the influence of the electromagnetic vacuum force on the behaviour of a model device based on materials, like germanium tellurides, that undergo fast and reversible metalinsulator transitions on passing from the crystalline to the amorphous phase. The calculations are performed at finite temperature and fully accounting for the behaviour of the material dielectric functions. The results show that the transition can be exploited to extend the distance and energy ranges under which the device can be operated without undergoing stiction phenomena. We discuss the approximation involved in adopting the Casimir expression in simulating nanoand micro- devices at finite temperature.
\end{abstract}

Introduction. - With the development of modern technology towards smaller structures an increasing attention has been addressed to the role of electromagnetic vacuum fluctuation forces (dispersion or van der Waals and Casimir forces) in micro- and nano-electromechanical systems [1-7]. These forces vary typically with the third or fourth inverse power of the distance between the surfaces of the interacting bodies and therefore can be very intense in the sub-micrometer regime. When the moving parts of a device come to such a close distance, dispersion forces affect the dynamics and the operation of the device and, under certain circumstances, they can cause adhesion or stiction between the surfaces, thus limiting the device lifetime. Devices actuated by Casimir force have been designed and demonstrated [8-10]. Since these forces depend upon the reflectivity, the size and the geometry of the interacting parts $[11,12]$, several possibilities can be explored to devise structures that exploit such dependence in order to improve the performance of existing devices or to design new types of devices [13].

In this paper we investigate the possibility of using materials, that undergo fast and reversible phase transitions, to extend the travel range of a device. Thermally induced phase changes between the amorphous and the polycrystalline state in a thin film have been observed since a (a)

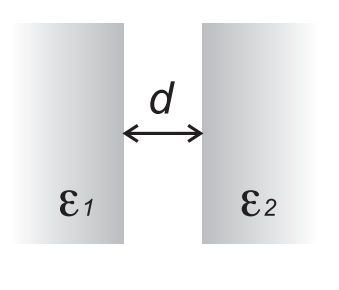

(b)

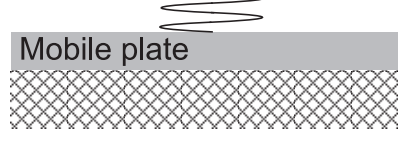

GeTe coating Substrate
Fig. 1: (a) Three layers model of equation (1); (b) Simple representation of the dispersion forces based device.

long time in tellurides containing Ge and $\mathrm{Sb}$ (GST materials) [14-21]. The transition is accompanied by significant changes in optical and transport properties, a feature that is exploited in optical data storage [22] and could be useful in new nanoscale memories [23, 24]. Previous theoretical studies have shown that metal insulator transitions may cause significant changes in the Casimir force behaviour $[25,26]$. In this paper we propose to use this transition to modify the force between the components of a simple device. We show that the locations of the extrema in the total energy potential curve of the device are 
displaced by the transition in such a way that the range of distances and the energy interval over which the device can be operated can be significantly modified.

The model device. - We consider an ideal actuator $[1,13]$ consisting of two parallel plates separated by a gap, with one plate fixed on a substrate and the other suspended by an elastic restoring force $F=-K x_{0} \delta$, where $x_{0}$ is the unactuated distance and $K$ is the spring constant. The stationary plate has a flat surface at $\delta=1$, while $\delta=0$ denotes the equilibrium position of the movable plate in the absence of dispersion forces and corresponds to the unstretched state of the spring. The attractive interaction between the plates of Fig. 1(a) is provided by the electromagnetic vacuum fluctuation force, whose expression in terms of the temperature $T$, the dielectric functions $\epsilon_{1}$ and $\epsilon_{2}$ and the inter-plate distance $d=(1-\delta) x_{0}$, is given by the Lifshitz formula $[27,28]$ :

$$
\begin{aligned}
F & =-\frac{A}{\pi \beta} \sum_{n=0}^{\infty} \int_{0}^{\infty} \gamma k d k\left[\frac{1-Q_{T M}\left(i \Omega_{n}\right)}{Q_{T M}\left(i \Omega_{n}\right)}+\right. \\
& \left.+\frac{1-Q_{T E}\left(i \Omega_{n}\right)}{Q_{T E}\left(i \Omega_{n}\right)}\right]
\end{aligned}
$$

here $A$ is the plate surface, $\beta=1 / k_{B} T, k_{B}$ is the Boltzmann constant, $\Omega_{n}=2 \pi n / \hbar \beta$ is the Matsubara frequency corresponding to the $n$-th thermal fluctuation mode, the prime on the summation indicates that the $n=0$ term is given half weight. We assume the plate size to be much larger than the separation distance. $Q_{T M}$ and $Q_{T E}$ refer to transverse magnetic (TM) and transverse electric (TE) modes respectively and are given by:

$$
\begin{gathered}
Q_{T M}\left(i \Omega_{n}\right)=1-\frac{\left(\epsilon_{1} \gamma-\gamma_{1}\right)\left(\epsilon_{2} \gamma-\gamma_{2}\right)}{\left(\epsilon_{1} \gamma+\gamma_{1}\right)\left(\epsilon_{2} \gamma+\gamma_{2}\right)} e^{-2 d \gamma} \\
Q_{T E}\left(i \Omega_{n}\right)=1-\frac{\left(\gamma-\gamma_{1}\right)\left(\gamma-\gamma_{2}\right)}{\left(\gamma+\gamma_{1}\right)\left(\gamma+\gamma_{2}\right)} e^{-2 d \gamma}
\end{gathered}
$$

with:

$$
\gamma_{i}^{2}=k^{2}+\frac{\Omega_{n}^{2}}{c^{2}} \epsilon_{i}\left(i \Omega_{n}\right) \quad \gamma^{2}=k^{2}+\frac{\Omega_{n}^{2}}{c^{2}}
$$

and the dielectric functions are evaluated at the frequency $i \Omega_{n}$ by means of the London transform:

$$
\epsilon(i \omega)=1+\frac{2}{\pi} \int_{0}^{\infty} \frac{\Im[\epsilon(y)]}{y^{2}+\omega^{2}} y d y
$$

where $\Im[\epsilon(y)]$ is the imaginary part of the dielectric function. In the calculation for the metallic phase we follow the prescription of ref. [29], i.e. we put the $n=0$ contribution of the TE modes equal zero. The behaviour of the force as a function of $d$ is non linear: at short distances it can be reproduced by a third inverse power of $d$ (van der Waals regime), but a single inverse power term does not reproduce its behaviour for $d$ values larger than a few tens of nanometers. As pointed out by several authors [30,31], mainly in connection with electrostatic actuators, there

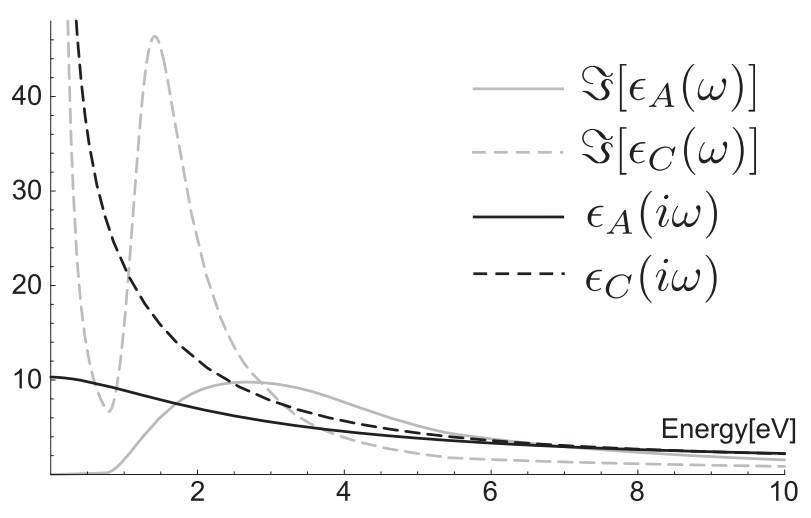

Fig. 2: Imaginary parts of amorphous and crystalline GeTe dielectric functions and their London transforms, adapted from ref. [36] as discussed in the text.

is an intrinsic instability in a device of this sort, which prevents the plates to be stably positioned over a large distance. The system is bistable, having a total potential energy with a local and an absolute minimum separated by a barrier. This limits the range of motion of the device and determines stiction at short distances between the plates $[1,2,4-6]$. We adopt the configuration illustrated in Fig. 1 (b) where both plates are made of telluride films deposited on proper substrates. For simplicity we assume that the thickness of the deposited films is larger than the distance between the plates (if the film size is comparable to the distance, the substrate is expected to affect the intensity of the force). This assumption can be removed by extending the expression of the Lifshitz force to deal with a five layer system $[13,32]$.

GeTe dielectric properties and the force. - To illustrate our model device, we focus our attention on GeTe, whose optical properties have been experimentally determined in both phases. It undergoes rapid transitions between polycrystalline and amorphous states under either optical or electrical excitations. According to the band theory in a perfect crystal rocksalt structure GeTe should exhibit a semiconducting behaviour. However the stable polycrystalline phases are characterized by large vacancy concentrations (typically $10^{20} \mathrm{~cm}^{-3}$ ) and local distortions $[33,34]$. Compared to other defects, that may be present, germanium vacancies have the lower formation energy [35]. GeTe thin films show a $p$-type electrical conductivity with a high carrier density of up to $5 \times 10^{20}$ $\mathrm{cm}^{-3}$. This density is consistent with the concentration of Ge vacant sites, i.e. the metallic conductivity is the consequence of the holes that are formed per single vacant site. In the optical properties direct evidence of the metallic behaviour is provided by the presence of Drudelike intraband absorption in crystalline phase [36] (see Fig. 2). On the other hand the amorphous phase has typical semiconductor properties, as illustrated by the behaviour of the imaginary part of the dielectric function, reported in the same figure. Conventional semiconductors, such as 


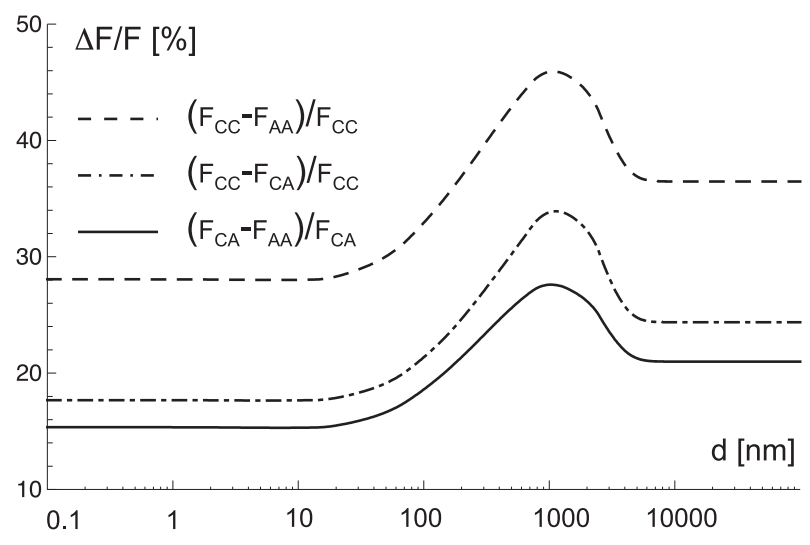

Fig. 3: Relative difference between $F_{C C}, F_{C A}$ and $F_{A A}$.

Si or GaAs, do not show such large differences in the optical properties as a consequence of the amorphous-crystal transition.

In Fig. 2 we display the calculated London transform for both phases. To obtain this curve we have reproduced the experimental data available in the range of frequencies $0.1 \div 5 \mathrm{eV}$ with a Drude-Lorentz model including both intra- and inter-band transitions in the metallic phase, and pure inter-band transitions in the amorphous phase. The values of the carrier density, the carrier effective mass, the relaxation time and the plasma frequency agree with those derived from various experimental studies [15-17]. It is seen that $\epsilon(i \omega)$ turns out to be very different in the two phases in almost all the range of frequencies. As a consequence of the intraband absorption, that is present in the metallic phase only, the curve for the polycrystalline phase for small $\omega$ takes much higher values than the one for the amorphous phase. Such differences over all the integration interval of equation (1) are expected to determine significant changes in the force. We can use equation (11) to calculate the force between two amorphous coated plates $F_{A A}$, two crystal coated plates $F_{C C}$, and the mixed configuration $F_{C A}$. In Fig. 3 we report, as a function of the separation distance, the relative variation of the dispersion force between the plates with respect to the configuration with both plates in the crystalline phase, when one or both plates undergo the transition from the crystalline to the amorphous phase. The curves have a similar behaviour with two ranges, at small and large distances, where the relative difference is constant, and a maximum in the range between $50 \mathrm{~nm}$ and $5 \mu \mathrm{m}$. The constant values are a consequence of the fact that the forces have identical behaviour as a function of the plate separation. At short distances the force can be approximated by [37]:

$$
F=-\frac{1}{4 \pi \beta d^{3}} \sum_{n=0}^{\infty} \sum_{m=1}^{\infty} \frac{1}{m^{3}}\left(\frac{\epsilon_{1}-1}{\epsilon_{1}+1}\right)^{m}\left(\frac{\epsilon_{2}-1}{\epsilon_{2}+1}\right)^{m}
$$

which gives a relative change of approximately $28 \%$, that does not depend upon the distance and the temperature. Notice that for the $n=0$ term of the Matsubara sum, the crystalline dielectric function diverges and:

$$
\frac{\epsilon-1}{\epsilon+1} \rightarrow 1 \quad \sum_{m=1}^{\infty} \frac{1}{m^{3}}=\zeta(3)
$$

where $\zeta(x)$ is the Riemann zeta function. On the other hand, at large distances within the present treatment of the temperature dependence of the Casimir force, the force between polycrystalline plates is simply given by $[29,38]^{1}$ :

$$
F_{C C} \simeq-\frac{\zeta(3)}{8 \pi \beta d^{3}}
$$

In the amorphous case the force for large $d$ cannot be written in a closed form. However it can be very well approximated by the $n=0$ term of the Matsubara sum

$$
F_{A A} \simeq-\frac{1}{8 \pi \beta d^{3}} L i_{3}\left[\left(\frac{\epsilon_{A}(0)-1}{\epsilon_{A}(0)+1}\right)^{2}\right]
$$

where $L i_{n}[x]$ is the polylogaritmic function of $n$-th order in the argument $x$. From these expressions one obtains a relative change of the order of $36 \%$, in agreement with the exact numerical results in Fig. 2

Tailoring the device performance. - Device instability is usually discussed in terms of a dimensionless parameter, like the ratio between the Casimir energy at the equilibrium distance and the elastic energy $[?, 1,13]$. This can be done conveniently when the force has a simple inverse power dependence with the separation distance. The analysis of the bifurcation diagrams as a function of the parameter allows one to draw conclusions about changes in the critical separation with the material dielectric properties. In our case the force as a function of the separation distance cannot be reproduced by a simple power law, so that we cannot adopt a single dimensionless parameter to study the device instability.

The behaviour of the device depends upon the spring constant $k$, the area $A$ of the plates and the unactuated distance $x_{0}$. The ratio $\Xi=A / k$ gives a measure of the relative importance of the vacuum and the elastic force, since they increase linearly with $A$ and $k$ respectively. According to the values attributed to these parameters one obtains different intervals of distances and energies of device operation at fixed $x_{0}$. To show the effects of the phase transition we give in Fig. 4 plots of the potential energy of the device for fixed $k$ and $A$ at $x_{0}$ values of the order of hundred nanometers. This choice corresponds to distances where the relative difference of the force varies significantly (see Fig. 3). As expected, on passing from the $C C$ to the $A A$ configuration the weakening of the vacuum force shifts the stable equilibrium position towards smaller $\delta$ values and the maximum at larger $\delta$. The displacement is more pronounced for low $\Xi$ i.e. soft spring

\footnotetext{
${ }^{1}$ Notice that this behaviour is different from the expression of the Casimir force at $T=0^{\circ} \mathrm{K}$ that for a perfect metal gives $F_{C C}=$ $-\frac{\hbar c \pi^{2}}{240 d^{4}}$. This formula has been frequently used in previous studies of the role of electromagnetic forces in stiction phenomena.
} 
(a)
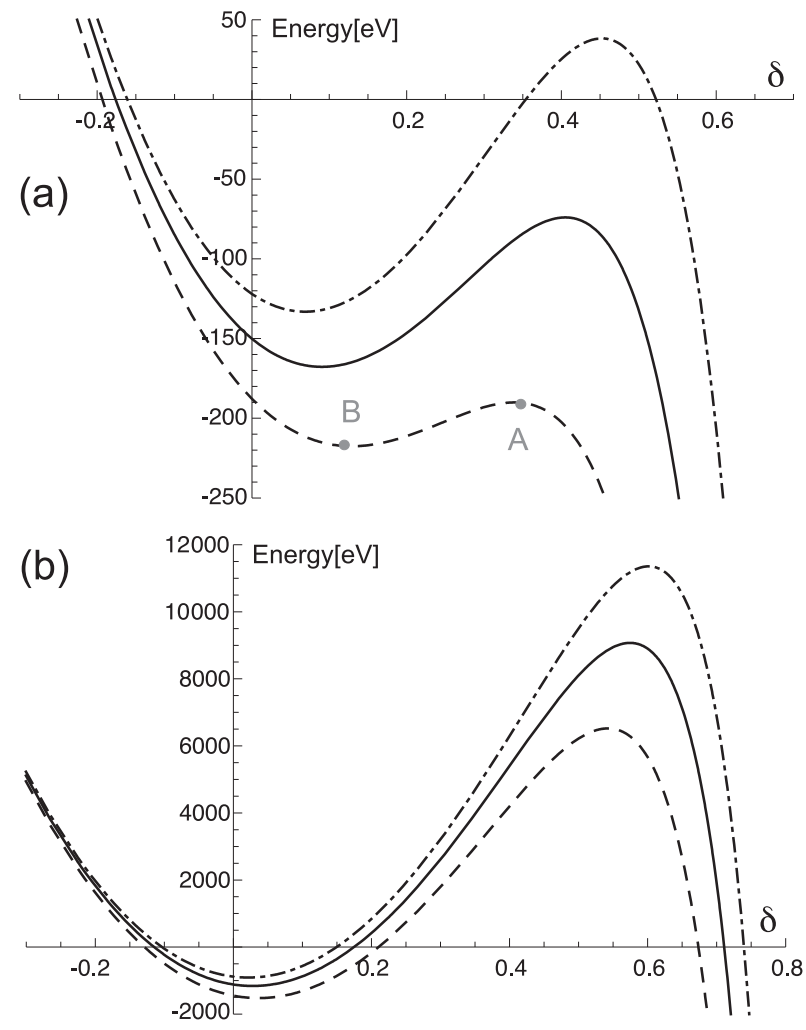

Fig. 4: Potential profiles for $C C$ configuration (dashed line), $C A$ configuration (continuous line) and $A A$ configuration (dotdashed line). In the (a) plot $x_{0}=100 \mathrm{~nm}, k=0.1 \mathrm{~N} / \mathrm{m}$ and $A=2 \cdot 10^{-10} \mathrm{~m}^{2}$ letters A and B refer to Fig. 55(a); in the (b) plot $x_{0}=200 \mathrm{~nm}, k=0.5 \mathrm{~N} / \mathrm{m}$ and $A=10^{-8} \mathrm{~m}^{2}$.

and small area (Fig. 4(a)), where it can be of the order of $80 \%$ and more, than in the case of large $\Xi$ plotted in Fig. 4(b), where the increase of the travel range is of the order of $10 \%$. The range of energies for which the device can be operated is modified in a similar way. These curves provide examples of the possibility of tuning in reversible way the device properties using the metal-insulator phase transition. Fig. 5 displays bifurcation diagrams of the device as a function of $\Xi$ for $x_{0}$ equal to $100 \mathrm{~nm}$ (Fig. 5 (a)) and $5 \mu \mathrm{m}$ (Fig. [5(b)) $[13,39,40]$ for the $C C, C A$ and $A A$ configurations. The curves have similar shapes, with a lower branch before the fold that corresponds to stable solutions while the upper branch indicates the unstable states. The $\delta$ values which allow to operate the device at a given value $\Xi^{\star}$ are those which lie within the intersections of the line $\Xi=\Xi^{\star}$ with the upper and the lower branch of the curves (points A and B in Fig. 5(a)). At the $\Xi$ value corresponding to the curve fold there is only one intersection, which gives the critical separation $\delta_{0}$. As expected, the crystalline-amorphous transition changes significantly the range of $\Xi$ parameters over which the device can work. Notice that $\delta_{0}$ varies between 0.21 and 0.22 . The critical value is expected to change when the functional dependence of the vacuum force with the plate separation is modified: for the $d^{-4}$ dependence typical of the Casimir
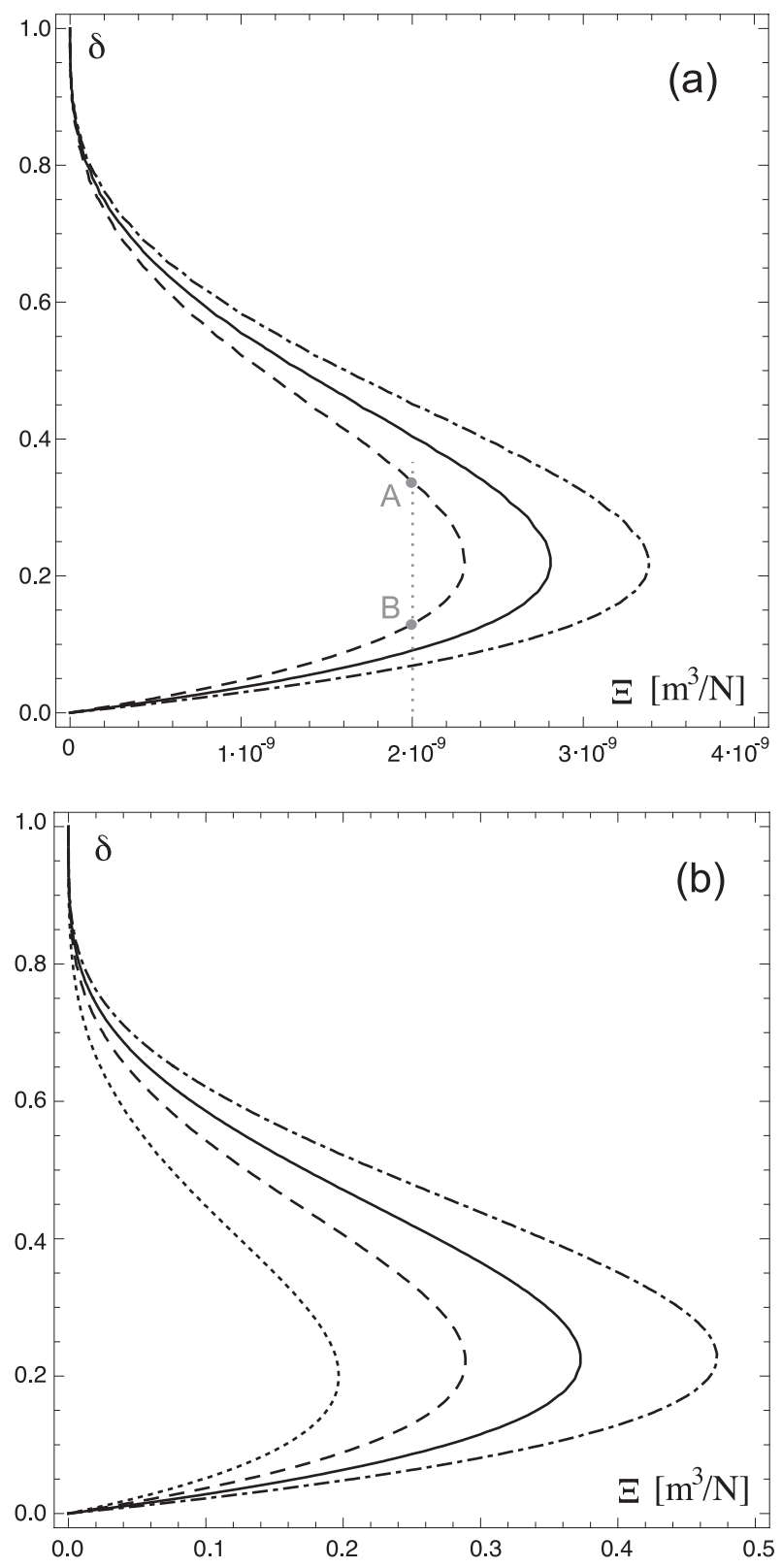

Fig. 5: Bifurcation diagrams for $C C$ configuration (dashed line), $C A$ configuration (continuous line) and $A A$ configuration (dot-dashed line). In the (a) plot $x_{0}=100 \mathrm{~nm}$, the dotted line

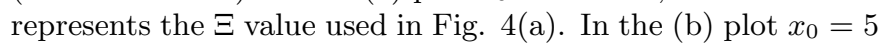
$\mu m$, the dotted line represents the force calculated using the $T=0^{\circ} K$ Casimir's force.

force $\delta_{0}=0.20$, while for the $d^{-3}$ behaviour, which is characteristic of the van der Waals interaction and of the large distance force at $T>0^{\circ} K, \delta_{0}=0.25[1,13]$. In the case illustrated in Fig. 5(a), i.e. $100 \mathrm{~nm}$ distance, the critical value deviates significantly from the third inverse power behaviour. The curves of the $5 \mu \mathrm{m}$ case show a similar behaviour. For the sake of comparison we have reported in Fig. 5(b) the curve corresponding to a pure Casimir interaction, which has been often adopted in similar device analysis. This force is appropriate to describe the case of 


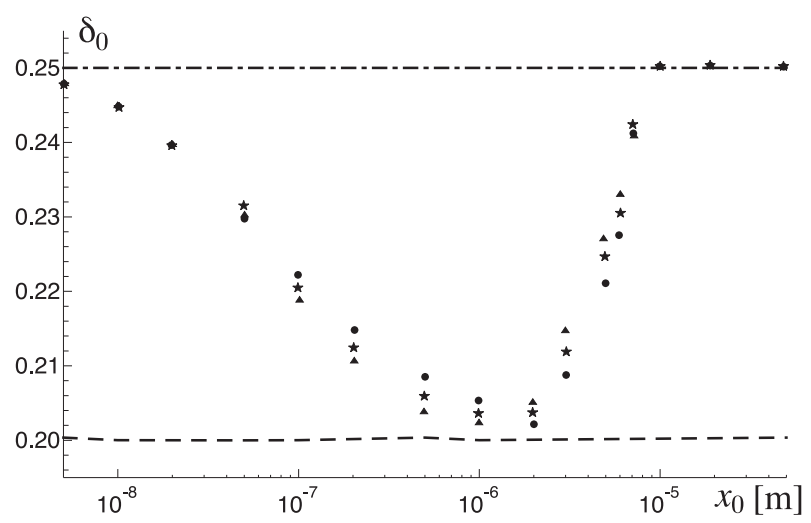

Fig. 6: $\delta_{0}$ as a function of $x_{0}$. Dashed line corresponds to the $T=0^{\circ} K$ Casimir's force, dot-dashed line is obtained in the small and large $d$ limits of equations (5) and (7). Dots represent the exact results for $F_{C C}$ (circles), $F_{A A}$ (triangles) and $F_{C A}$ (stars).

metallic plate at large distances for $T=0^{\circ} K$. Comparison with the results for our $C C$ configuration shows that the ranges of $\Xi$ and $\delta$ values are considerably underestimated by this interaction. Notice that the vacuum force at such distance cannot yet be described by a third inverse power law, as expected from equation (17) at $T>0^{\circ} \mathrm{K}$. This is most clearly seen by the critical $\delta_{0}$ value which is smaller than 0.25 .

To better illustrate how the separation distance dependence of the vacuum force can affect the device performance, we plot in Fig. 6] the critical value as a function of the unactuated distance. It is seen that $\delta_{0}$ varies between 0.25 at very short (less than $10 \mathrm{~nm}$ ) and very large (more than $10 \mu \mathrm{m}$ ) distances and a minimum slightly larger than 0.20. The minimum occurs at different distances in the three configurations and it is found at smaller $x_{0}$ for the $A A$ case. It is clear from this curve that simulating device behaviour on the basis of simple inverse power law forces does not allow to get realistic results.

Conclusions. - We have shown how the metalinsulator transition in germanium tellurides can be exploited in a simple device to modify its performance and the conditions under which it can be used. The choice of the appropriate values for the parameters entering into our model may depend upon a number of factor, like the film quality [41], the possibility of controlling the kinetics of the phase transition, the mechanical properties of the components, the surface roughness [42] etc. which have to be clarified in order to plan the realization of a specific device. On the theoretical side two aspects have to be further investigated. The first concerns the role of the film size in determining the change in the vacuum force: this can be carried out along the lines of previous studies $[13,32]$ with the purpose of determining the minimum thickness that allows to detect significant vacuum force variations. The second has to do with the effects of the finite plate area, which in the metal case may be needed to avoid inconsistencies related to the Drude description of the intraband absorption [43].

\section{$* * *$}

The authors want to acknowledge Stephan Kremers (RWTH Aachen University) for the material he shared with them. AB is also grateful to Erio Tosatti (ICTP/SISSA Trieste) and Wojciech Welnic (LSI Ecole Polytechnique Palaiseau) for helpful discussions. AB thanks CINECA Consorzio Interuniversitario for funding his $\mathrm{PhD}$ fellowship.

\section{REFERENCES}

[1] F.M. Serry, D. Walliser, and G.J. Maclay. J. Appl. Phys., 84:2501, 1998.

[2] F.M. Serry, D. Walliser, and G.J. Maclay. J. Microelectromechanical Syst., 4:193, 1995.

[3] E. Buks and M.L. Roukes. Europhys. Lett., 54:220, 2001.

[4] E. Buks and M.L. Roukes. Phys. Rev. B, 63:033402, 2001.

[5] H.J. De los Santos. Proc. IEEE, 91:1907, 2003.

[6] F.W. Delrio, M.P. De Boer, J.A. Knapp, P.J. Clews E.D. Reedy jr, and M.L. Dunn. Nature Mat., 4:629, 2005.

[7] R.C. Batra, M. Porfiri, and D. Spinello. Europhys.Lett., $77: 20010,2007$.

[8] H.B. Chan, V.A. Aksyuk, R.N. Kleiman, D.J. Bishop, and F. Capasso. Science, 291:1941, 2001.

[9] H.B. Chan, V.A. Aksyuk, R.N. Kleiman, D.J. Bishop, and F. Capasso. Phys.Rev.Lett., 87:211801, 2001.

[10] A. Ashourvan, M. Miri, and R. Golestanian. Phys.Rev.Lett., 98:140801, 2007.

[11] D. Iannuzzi, M. Lisanti, and F. Capasso. Proc. Nat. Ac. Sci. USA, 101:4019, 2004

[12] F. Chen, G.L. Klimchitskaya, V.M. Mostepanenko, and U. Mohideen. Phys.Rev.B, 76:035338, 2007.

[13] J. Barcenas, L. Reyes, and R. Esquivel-Sirvent. Appl. Phys. Lett., 87:263106, 2005.

[14] S.R. Ovshinsky. Phys.Rev.Lett., 21:1450, 1968.

[15] K.L. Chopra and S.K. Bahl. J.Appl.Phys., 40:4171, 1969.

[16] S.K. Bahl and K.L. Chopra. J.Appl.Phys., 40:4940, 1969.

[17] S.K. Bahl and K.L. Chopra. J.Appl.Phys., 41:2196, 1970.

[18] N. Yamada, E. Ohno, K. Nischiuchi, N. Akahira, and M. Takao. J.Appl.Phys., 69:2849, 1991.

[19] N. Yamada. MRS Bull., 21:48, 1996.

[20] M. Libera and M. Chen. J.Appl.Phys., 73:2272, 1993.

[21] B.S. Lee, J.R. Abelson, S.G. Bishop, D.H. Kang, B. Cheong, and K.B. Kim. J.Appl.Phys., 97:093509, 2005.

[22] T. Ohta. J. Optoelectr. Adv. Mat., 3:609, 2001.

[23] M.H.R. Lankhorst, B.W.S.M.M. Ketelaars, and R.M. Wolters. Nature Mat., 4:347, 2005.

[24] M. Wuttig. Nature Mat., 4:267, 2005.

[25] I. Pirozhenko and A. Lambrecht. Phys.Rev.A, 77:013811, 2008.

[26] R. Esquivel-Sirvent. Phys. Rev. A, 77:042107, 2008.

[27] E.M. Lifshitz. Sov. Phys. JEPT, 2:73, 1956.

[28] I.E. Dzyaloshinskii, E.M. Lifshitz, and L.P. Pitaevskii. Adv. Phys., 10:165, 1958.

[29] K.A. Milton. J. Phys. A: Math. Gen., 37:R209, 2004.

[30] K.L. Ekinci and M.L. Roukes. Rev. Sci. Instrum., 76:061101, 2005. 
[31] Wen-Ming Zhang, Guang Meng, and Di Chen. Sensors, 7:760, 2007.

[32] F. Zhou and L. Spruch. Phys. Rev. A, 52:297, 1995.

[33] W. Welnic, A. Pamungkas, R. Detemple, C. Steimer, S. Blugel, and M. Wuttig. Nature Mat., 5:56, 2005.

[34] M. Wuttig, D. Lusebrink, D. Wamwangi, W. Welnic, M. Gilleben, and R. Dreonskowski. Nature Mat., 6:122, 2007.

[35] A.H. Edwards, A.C. Pineda, P.A. Schultz, M.G. Martin, A.P. Tompson, H.P. Hjalmarson, and C.J. Umrigar. Phys. Rev. B, 73:045210, 2006.

[36] W. Welnic, S. Botti, L. Reining, and M. Wuttig. Phys.Rev.Lett., 98:236403, 2007.

[37] U. Hartmann. Phys. Rev. B, 42:1541, 1990.

[38] J.S. Høye, I. Brevik, J.B. Aarseth, and K.A. Milton. Phys. Rev. E, 67:056116, 2003.

[39] A. Pelesko. SIAM J. Appl. Math., 62:888, 2002.

[40] Wen-Hui Lin and Ya-Pu Zhao. Chaos,solitons and fractals, 23:1777, 2005.

[41] I. Pirozhenko, A. Lambrecht, and V.B.Svetovoy. New J. Phys., 8:238, 2006.

[42] G. Palasantzas and J.Th. DeHosson. Phys. Rev. B, 72:121409, 2005.

[43] B. Geyer, G.L. Klimchitskaya, and V.M. Mostepanenko. J.Phys.A: Math.Gen., 40:13485, 2007. 